\title{
La langue oudmourte en Oudmourtie entre 1990 et 2013 : état des lieux et perspectives
}

Udmurt Language in Udmurtia between 1990 and 2013: Assessment and Prospects

\section{Marie Casen}

\section{(2) OpenEdition}

\section{Journals}

Édition électronique

URL : https://journals.openedition.org/efo/1693

DOI : $10.4000 /$ efo. 1693

ISSN : 2275-1947

\section{Éditeur}

INALCO

Édition imprimée

Date de publication : 1 décembre 2013

ISBN : 978-2-343-04446-0

ISSN : 0071-2051

\section{Référence électronique}

Marie Casen, "La langue oudmourte en Oudmourtie entre 1990 et 2013 : état des lieux et perspectives », Études finno-ougriennes [En ligne], 45 | 2013, mis en ligne le 26 janvier 2015, consulté le 08 juillet 2021. URL : http://journals.openedition.org/efo/1693 ; DOI : https://doi.org/10.4000/efo. 1693

Ce document a été généré automatiquement le 8 juillet 2021.

\section{(c) (1) \&}

Études finno-ougriennes est mis à disposition selon les termes de la Licence Creative Commons Attribution - Pas d'Utilisation Commerciale 4.0 International. 


\section{La langue oudmourte en Oudmourtie entre 1990 et 2013 : état des lieux et perspectives}

Udmurt Language in Udmurtia between 1990 and 2013: Assessment and

Prospects

Marie Casen

$1 \mathrm{Au}$ XIX ${ }^{e}$ siècle, un ethnographe originaire de Kazan, I. N. Smirnov, prédisait la disparition des Oudmourtes pour une centaine d'années plus tard (Nikitina, 2013). Au $\mathrm{xxI}^{\mathrm{e}}$ siècle, les Oudmourtes sont toujours là, mais la langue figure dans l'atlas des langues en danger de l'UNESCO ${ }^{1}$. Cet article traite des évolutions qui se sont produites pour la langue oudmourte en Oudmourtie ${ }^{2}$ depuis la fin de la période soviétique. Une première partie présente les aspects liés à l'action des pouvoirs publics depuis 1990 : la législation, les programmes de sauvegarde linguistique et les démarches civiques pouvant être accomplies en oudmourte. Une deuxième partie montre les moyens mis en œuvre par les acteurs privés (les organisations politiques, artistiques et les enseignes commerciales), qui ont pour effet de suppléer aux institutions de représentation des langues minoritaires en augmentant la visibilité de l'oudmourte dans l'espace public. Une troisième partie interroge les fonctions de la langue oudmourte en 2013. Cet article repose en majeure partie sur des travaux de terrain effectués en 2008 et en 2013.

D'après les résultats du dernier recensement de la population de Russie en $2010^{3}$, les locuteurs de l'Oudmourte sont 324 338, soit $29,9 \%$ de moins que lors du recensement de 20024. Cette perte est comparable à celle des peuples finno-ougriens voisins : en 2010, on comptait $29,7 \%$ de locuteurs mordves (erza et mokcha) en moins par rapport à 2002, 28,1 \% de locuteurs du komi (zyriène et permiak) en moins et 20,3 \% de locuteurs du mari (des plaines et des collines) en moins. Cette situation illustre le phénomène d'assimilation linguistique des peuples allogènes à la population russe. Les chiffres des recensements montrent que les langues finno-ougriennes sont de moins en moins parlées: à la disparition des générations les plus anciennes, pour qui 
l'oudmourte était la langue de communication au village, et à l'exode rural inévitable, s'ajoute le désintérêt des générations les plus jeunes, qui arguent du manque d'attractivité et d'utilité pratique de la langue oudmourte dans le monde moderne (Casen 2008). Néanmoins, au-delà des chiffres, l'observation sur place des occasions de communication en oudmourte et l'apparition de signes visibles de son usage dans le domaine public permettent de préciser et de nuancer ce panorama statistique négatif.

3 En dehors de la période de reconnaissance des minorités linguistiques de l'Union soviétique dans les années 1920, c'est à partir des années 1990 que la langue oudmourte devient un objet sur lequel on légifère : elle devient, aux côtés du russe, la langue officielle de la République. Ce statut devrait avoir pour conséquence la plus évidente une importante visibilité de la langue dans l'espace public, avec notamment des panneaux routiers, des noms de rues et des enseignes officielles bilingues (Zamjatin, Pasanen, Saarikivi 2012), et la possibilité pour les locuteurs de l'oudmourte d'avoir accès aux services municipaux en oudmourte (formulaires, documents juridiques, dont la Constitution, communication avec les fonctionnaires). Que constate-t-on dans ces domaines? D'une part, la langue oudmourte est globalement peu visible dans l'espace public (sauf dans la capitale de la République, Iževsk, où les enseignes des ministères et des bâtiments officiels sont en russe et en oudmourte), et les services publics ne sont pas disponibles en langue oudmourte, faute de formation linguistique des personnels. D'autre part, les avancées sont lentes à se mettre en place : la Constitution de 1995 a été traduite en oudmourte dix ans après, et un seul ministère (celui de la politique nationale, chargé de représenter les peuples de l'Oudmourtie) a un site Internet bilingue - et ce, seulement depuis 2012. Enfin, les évolutions sont inégales à l'échelle de l'Oudmourtie. Dans les raïons où la part de la population oudmourte par rapport à la population totale est la plus importante (Alnaši, Malopurga, Možga et Uva), on a largement accès aux services publics en oudmourte - tant qu'il ne s'agit pas de formulaires officiels - puisque c'est la langue de la plupart des habitants. En revanche, à Iževsk, c'est seulement depuis cette année qu'il est possible de voir prononcer sa cérémonie de mariage en oudmourte à la mairie ${ }^{5}$ à condition de l'avoir demandé à temps, puisqu'il faut trouver un fonctionnaire qui connaisse l'oudmourte.

4 Ce n'est pas seulement le statut officiel de l'oudmourte qui garantit, en théorie, son développement, mais aussi son statut de langue d'un peuple de Russie (Casen 2013). En effet, selon l'article 68 de la Constitution russe de 1993, "la Fédération de Russie garantit à tous ses peuples le droit de préserver leur langue maternelle, de créer les conditions pour son enseignement et son développement "; de même, en vertu des articles 10, 11 et 12 de la loi fédérale sur l'Autonomie nationale et culturelle du 17 juin 1996, « les citoyens de la Fédération ont droit à une éducation dans leur langue maternelle ». C'est donc en particulier dans le domaine éducatif que l'aménagement linguistique de l'oudmourte devrait être le plus avancé, comme c'est le cas au Tatarstan voisin (Marcquardt 2012). En réalité, le dispositif d'enseignement de l'oudmourte à Iževsk n'a pas évolué depuis les années 1990, il s'est même dégradé en 2007, avec l'adoption de la loi fédérale (numéro 309) sur l'Éducation, qui viole les principes constitutionnels énoncés plus haut en interdisant les évaluations dans une autre langue que le russe, ce qui exclut encore un peu plus la composante oudmourte de l'éducation publique. Actuellement, dans certains villages oudmourtes, il $\mathrm{y}$ a une crèche où l'oudmourte est la langue de communication, et parfois encore une école primaire où l'oudmourte est enseigné en tant que matière. À Iževsk, l'école Kuzebaj Gerd dispense des cours d'oudmourte à tous les élèves, et huit gymnasium proposent des cours de 
langue et culture oudmourtes en option. L'université propose une filière de philologie oudmourte, et l'Institut d'histoire, de langue et de littérature accueille les chercheurs en langue et culture oudmourtes, mais la langue reste absente de toutes les autres filières ${ }^{6}$, dont celles qui préparent à un avenir commercial, scientifique ou politique.

Un deuxième niveau institutionnel consiste en l'action menée par le ministère de la Politique nationale (Nikitina, 2013), chargé d'élaborer et de conduire des programmes de sauvegarde de la langue oudmourte, afin de la populariser et de favoriser le bilinguisme. Le premier programme couvrait la période allant de 2005 à 2009 ; il était destiné à permettre la publication d'ouvrages dans tous les genres en langue oudmourte et à renforcer la diffusion des médias (presse locale et nationale, émissions télévisées et radiophoniques). Les résultats de ce programme n'ont pas été publiés, mais le ministère a effectué une enquête à mi-parcours, qui a mis en évidence l'échec partiel de ce programme : entre 2005 et 2007, seulement 19 titres ont été publiés en langue oudmourte, pour un total de 50000 exemplaires. Le programme actuel (2010-2014) est ciblé sur les Oudmourtes les plus jeunes : il vise à favoriser la diffusion d'ouvrages et d'émissions télévisées destinés aux enfants. Les résultats de ce programme ne sont pas publiés; cependant, on ne peut que constater le manque de points de vente de journaux ou de livres en oudmourte, aussi bien en ville qu'à la campagne, et il est peu probable que la diffusion de ces ouvrages pour les enfants soit effectuée à vaste échelle par correspondance. En ce qui concerne la télévision, on la trouve partout, et la chaîne nationale Moja Udmurtija diffuse une partie de ses programmes en oudmourte; il existe effectivement deux émissions destinées aux enfants $^{7}$, mais l'évolution la plus notable concerne les émissions destinées aux jeunes : une émission d'analyse de l'information, Maly ke šuono, une émission qui présente des lieux intéressants et inédits en Oudmourtie (notamment des villages) et sous-titrée en russe, Mon Egit, et une émission culinaire de téléréalité très largement suivie par les Oudmourtes, toutes générations confondues, une sorte de Top Chef oudmourte. En ce qui concerne la radio, elle est accessible partout, mais la plupart des programmes sont en russe; ce sont surtout les personnes âgées qui l'écoutent ${ }^{8}$. Elle est de plus en plus supplantée par la télévision de la même compagnie publique (Moja Udmurtija) dont le site Internet permet la retransmission à la carte des émissions en oudmourte, ce qui n'est pas le cas pour la radio.

6 En vingt-trois ans, les lois et les projets institutionnels n'ont donc eu que peu d'effet sur la présence de la langue oudmourte dans l'enseignement, sur son accessibilité ou sa visibilité dans l'espace public.

7 Pourtant, depuis 2010, on peut constater une évolution sensible de la visibilité de l'oudmourte et de son accessibilité dans les zones où il n'était pas présent avant (en particulier à Iževsk), sous l'impulsion d'acteurs non institutionnels et/ou étrangers. On observe un transfert des compétences des organisations officielles vers des groupes non liés aux institutions: une partie des actions de revitalisation linguistique normalement dévolues à l'État est de facto prise en charge par de jeunes Oudmourtes bénévoles, souvent citadins et ayant appris l'oudmourte à l'école ${ }^{9}$, organisés en associations ou en groupes de promotion, et dont l'outil principal est l'Internet. Voici quelques exemples de ce transfert de compétences :

8 La modernisation de la langue, son adaptation au monde moderne par la création de néologismes, était menée depuis 1995 par une commission officielle composée de chercheurs et de personnalités politiques. Une fois accomplie sa première mission, qui 
était de traduire la Constitution, la commission n'a pas continué activement son travail ${ }^{10}$. Udmurtlyk, un groupe visible uniquement sur Internet, sans existence officielle et pourtant très populaire parmi les générations les plus jeunes, utilise les réseaux sociaux ${ }^{11}$ pour poursuivre ce travail par un système de vote ${ }^{12}$. Le choix des internautes est transmis aux principaux médias (télévision, radio, journaux) afin qu'ils s'en inspirent.

Conscients de la nécessité de revitalisation linguistique et d'élargissement des fonctions de communication de leur langue, ces jeunes Oudmourtes, dont environ la moitié n'a pas l'oudmourte pour langue maternelle, organisent un enseignement de langue destiné aux non-oudmourtophones, gratuit, en ligne ${ }^{13}$ (quotidien) et en présentiel (par des sessions de cours intensif). En parallèle, ils accroissent la diversité de l'offre linguistique aux oudmourtophones en écrivant de nombreux textes de genres variés ${ }^{14}$ sur les forums, les blogs et les sites Internet en langue oudmourte, ou encore dans les journaux ${ }^{15}$. La production de textes en langue oudmourte et l'invention de néologismes posent la question de la standardisation de la langue oudmourte, sujet apparemment abandonné par les instances officielles de représentation des Oudmourtes. La normalisation de l'oudmourte est un projet décisif, parce que les variétés dialectales sont nombreuses, rurales et en voie de disparition, et que la langue standard actuelle est une langue artificielle, citadine, incomplète et difficilement lisible, même pour les générations les plus jeunes. L'émergence d'une langue standard est une garantie de visibilité et de diffusion importante de la langue. Ce processus suppose notamment de définir « les limites acceptables des emprunts lexicaux et des néologismes, en résolvant le problème de la juste proportion entre la langue littéraire et les formes dialectales " (Šabaev, Šilov, Denisenko 2009, p. 98), et l'on peut se demander si des locuteurs, qui en majorité n'ont pas l'oudmourte comme langue maternelle, ni les moyens de développer des programmes à grande échelle pour diffuser la langue standard, sont à même d'accomplir ce travail.

Un autre aspect de ce transfert de compétences consiste en la diffusion des ouvrages en langue oudmourte. Les avancées dans ce domaine sont nombreuses et notables: la bibliothèque nationale de Finlande a créé un blog, Fenno-Ugrica ${ }^{16}$, afin de numériser et mettre en ligne les ouvrages en langues finno-ougriennes. Il s'agit du projet Uralica, financé par la Fondation Kone et mené en coopération avec les bibliothèques de Russie, dont la bibliothèque nationale d'Oudmourtie, qui vient de procéder à la numérisation d'environ 130 ouvrages en langue oudmourte. Depuis 2012, plus de 153 ouvrages en langues finno-ougriennes (dont des dictionnaires) ont été mis en ligne, ainsi que 5098 journaux (dont certains datant des années 1920 et 1930). À côté de ce projet à grande échelle, il existe des initiatives locales qui pallient le manque de lieux de diffusion par l'usage de l'Internet: le groupe de réflexion Muš mène depuis plusieurs années une entreprise de numérisation des ouvrages en langue oudmourte des années 1920 et 1930 ; par ailleurs, le supplément artistique de l'Udmurt Dunne, Dart, le magazine bilingue Invožo, et le journal des Oudmourtes de Bachkirie Ošmes, entre autres, sont accessibles en ligne.

11 Enfin, en termes de visibilité de l'oudmourte dans l'espace public, ce sont les activités non institutionnelles et issues de la pénétration des codes et des modes de vie occidentaux en Russie qui ont le plus modifié le paysage en Oudmourtie, en particulier dans les villes. D'une part, le positionnement d'acteurs économiques occidentaux sur le marché russe crée des possibilités inédites de visibilité de la langue oudmourte. Ainsi, 
la stratégie de la chaîne de hard discount " Metro » utilise la langue locale comme outil marketing : dans le magasin d'Iževsk, toutes les étiquettes sont bilingues. D'autre part, les nouvelles manifestations culturelles et politiques liées à l'ouverture sur le monde de la Russie postsoviétique rendent possible une expression publique en oudmourte : par exemple, en réaction aux nominations farfelues, effectuées par le député Envil Kasimov, de personnalités au titre de citoyens d'honneur d'Oudmourtie (Emir Kusturica, Steve Jobs, Gérard Depardieu), une poignée de personnes ont demandé, en oudmourte, à bénéficier de ce titre normalement destiné aux individus ayant été utiles au développement de l'Oudmourtie (photo 1). De même, à Iževsk, lors des rassemblements de contestation faisant suite à l'élection de V. Poutine en mars 2012, on a pu voir des pancartes en oudmourte brandies par les manifestants pour plus de justice, non pour la cause oudmourte, mais en tant que citoyens d'Oudmourtie ${ }^{17}$; en outre, tags et graffitis en oudmourte sont de plus en plus courants sur les murs de la ville (photos 2 et 3 ).

Photo 1 : «Envil, écris-moi, je suis aussi citoyen d'honneur oudmourte ! »

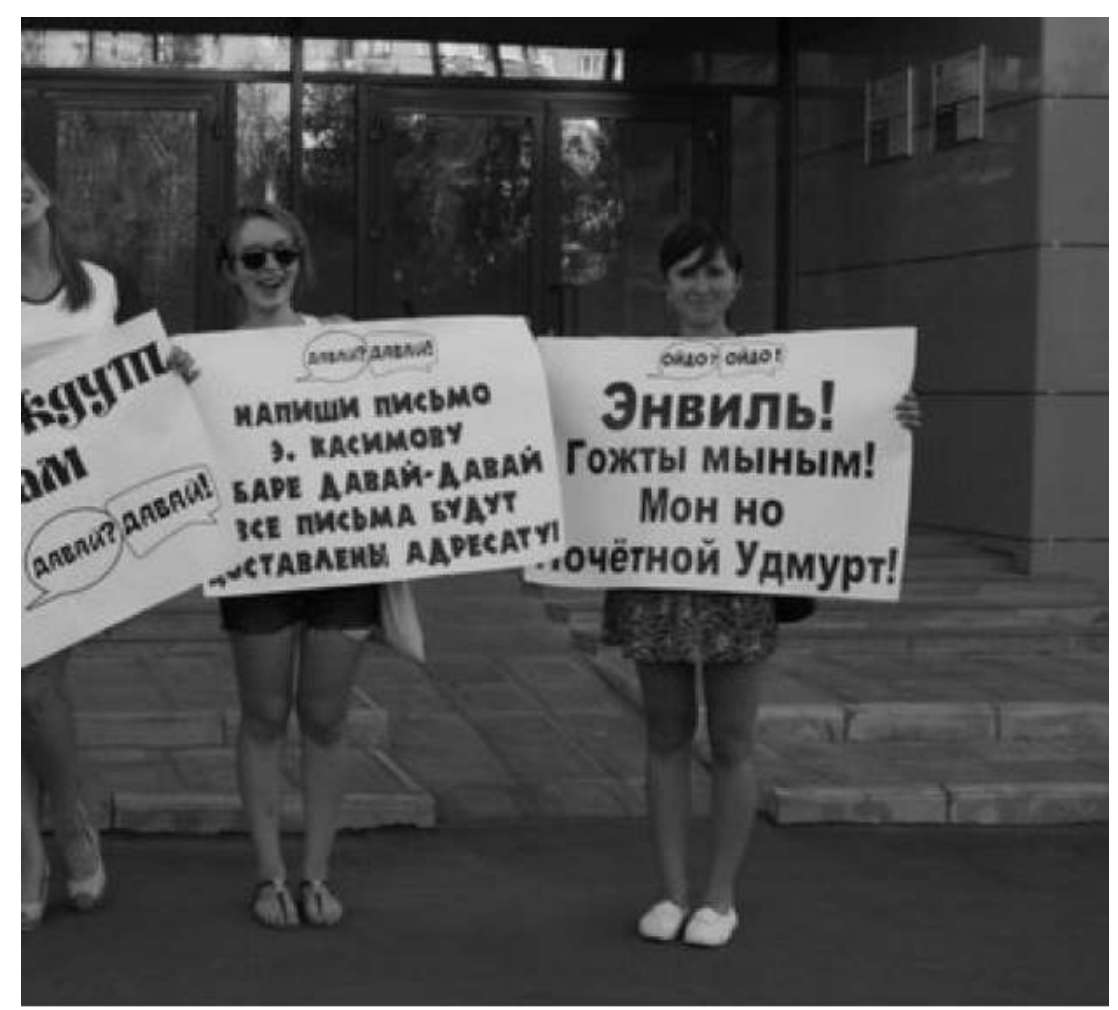


Photo 2 : «Dans le cosmos il y a des dieux qui regardent »

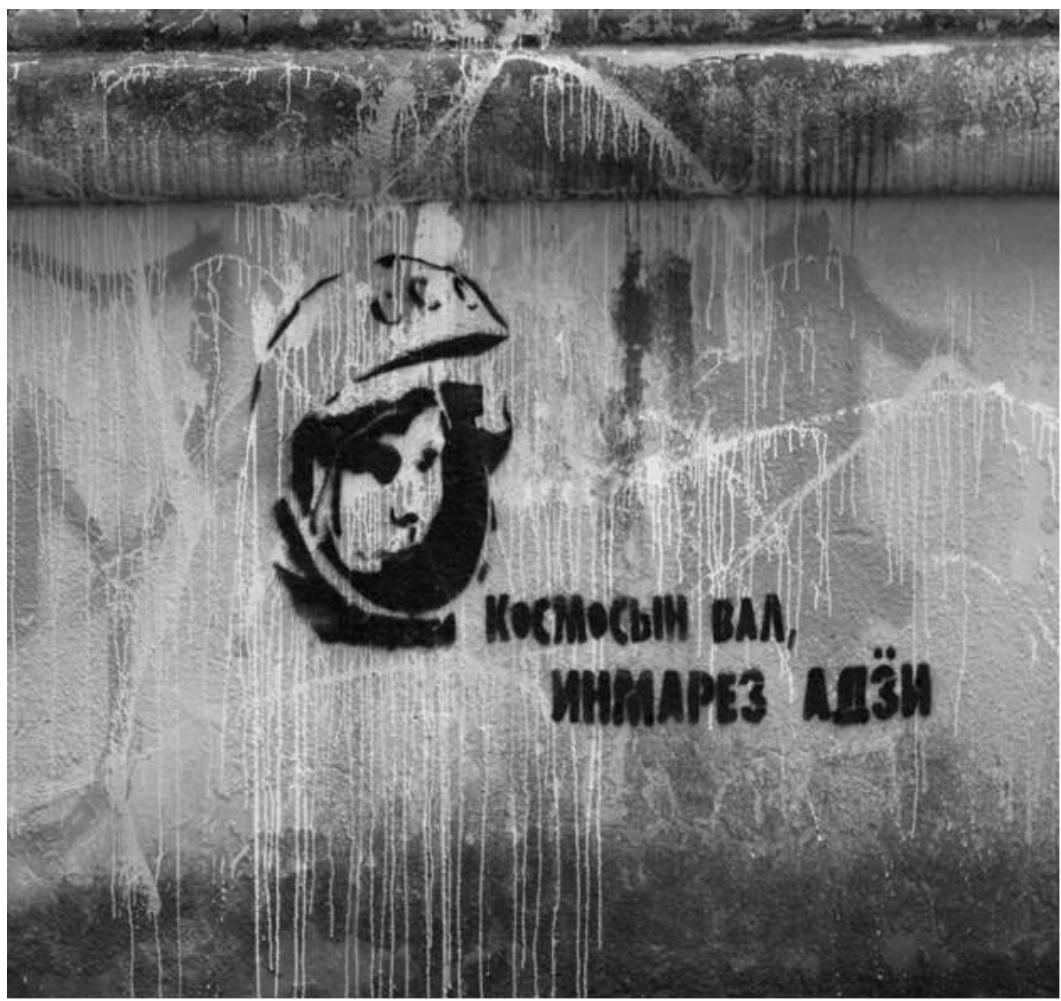

Photo 3 : «Parle correctement ! »

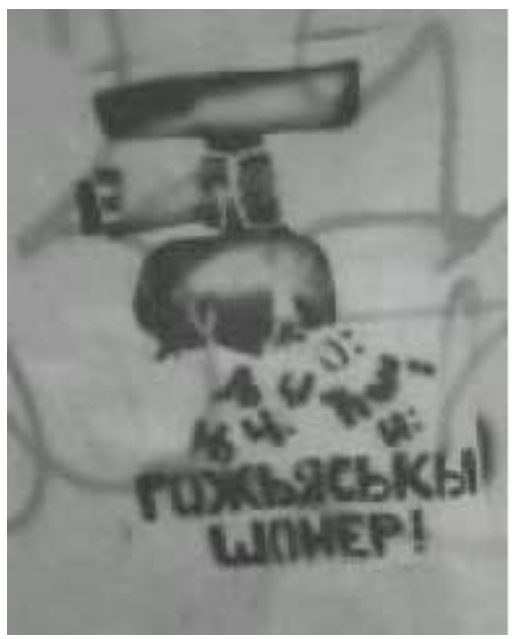

12 Peut-être le changement des mentalités et le positionnement politique des acteurs de la revitalisation de la langue oudmourte en sont-ils à l'origine; ou l'absence quasi générale, quoiqu'explicable, de la langue oudmourte à Iževsk lui confère-t-elle un aspect de dissidence, cher aux acteurs des mouvements contestataires qui se manifestent dans la Russie actuelle.

13 Les occasions d'entendre l'oudmourte sont rares, à l'exception des zones où vivent ses locuteurs: dans les villages et aux alentours de l'université à Iževsk; néanmoins, dans certaines situations de la vie quotidienne (achat d'un téléphone, de nourriture, recherche de documents aux archives nationales, demande d'informations, etc.), on s'aperçoit sans difficulté, en posant la question, qu'on a affaire à des Oudmourtes, 
parfois oudmourtophones ${ }^{18}$, ce qui semble montrer que les mentalités ont changé et qu'il n'est plus ni honteux ni déplacé de se présenter comme oudmourte. De même, dans le domaine artistique, la chanson oudmourte est de plus en plus populaire, en particulier hors des frontières de la Russie, et présente dans des genres variés. Cette tendance est illustrée notamment par le succès récent de groupes musicaux oudmourtes sur la scène internationale: les babouchkas de Buranovo ont remporté la seconde place au concours de l'Eurovision à Bakou en 2012; le groupe de rock oudmourte Silent Woo Goore a trouvé un public en Finlande, et la notoriété du chanteur Ullapalla Boy, qui réalise des versions oudmourtes ${ }^{19}$ des plus grands tubes planétaires actuels, est grandissante à travers le monde, grâce aux réseaux sociaux.

$\mathrm{Au}$ regard des évolutions précitées, on peut se demander quelles fonctions occupe la langue oudmourte en Oudmourtie aujourd'hui.

D'abord, la fonction identitaire de la langue oudmourte semble de moins en moins déterminante, comme l'indiquent les chiffres des recensements. En 2002, 173569 personnes ont déclaré être Oudmourtes, mais non-locuteurs de l'oudmourte. On peut se dire Oudmourte sans parler la langue. Cette tendance se confirme en 2010, puisque cette fois 227961 personnes (un tiers de plus) se sont déclarées Oudmourtes sans maîtriser cette langue. On peut se demander quels sont les processus d'identification en cours parmi les Oudmourtes non-locuteurs de cette langue, pour lesquels l'appartenance à un groupe est bien identifiée, mais fondée sur des critères non-linguistiques.

Ensuite, une chercheuse oudmourte, Svetlana Edygarova, a souligné que l'oudmourte était la langue de communication pour une minorité des locuteurs qui déclarent la maitriser (Edygarova 2012). En dehors des zones où les Oudmourtes représentent la population majoritaire, ce qui rend possible l'usage de l'oudmourte pour la communication entre des personnes qui ne se connaissent pas, l'oudmourte est le plus souvent parlé dans le cercle familial et cantonné à un usage domestique. L'oudmourte des locuteurs natifs les plus jeunes risque donc de s'appauvrir ${ }^{20}$, et paradoxalement, ce sont les Oudmourtophones non-natifs qui ont le plus accès aux nuances de la langue, qui la comprennent le mieux - en tout cas pour la langue standard, qui est la variété utilisée par les médias et qu'utilisent les locuteurs les plus soucieux de garantir l'avenir de la langue oudmourte en œuvrant à sa standardisation et à sa diffusion.

17 La fonction de transmission culturelle de la langue, assurée surtout par les grandsparents en direction des petits-enfants, à partir des années 1990 et dans la première moitié des années 2000 (Guboglo, Smirnova 2002), diminue avec la disparition naturelle des personnes âgées. On peut supposer que cette perte sera compensée par les nouveaux parents oudmourtophones, mais il n'existe pas de chiffre permettant d'évaluer dans quelle mesure. Quoi qu'il en soit, une partie des oudmourtophones ne le sont pas de langue maternelle et n'ont pas de lien originel avec la campagne : on peut donc penser que le contenu culturel qu'ils transmettront sera sensiblement différent de celui des générations précédentes.

18 On sait que dans certaines situations, la langue oudmourte permet aux Oudmourtes de manifester leur appartenance à un groupe ethnique tout en se distinguant des autres groupes (Casen 2008). Il semble que la fonction distinctive de l'oudmourte mérite une analyse plus fouillée. En effet, même si l'usage de l'oudmourte dans un contexte non spécifiquement oudmourte manifeste une appartenance à un groupe ethnique, elle ne semble pas être une revendication ethnique qui aurait pour origine une volonté de se 
distinguer des Russes ou des Tatars. Par exemple, le succès de musiciens et d'artistes oudmourtes sur la scène internationale, en particulier dans les pays finno-ougriens d'Europe, ou encore la représentation des chercheurs oudmourtes à l'étranger, donnent plutôt l'impression que la langue oudmourte a sa place (au moins en tant qu'outil artistique et sujet de recherche) dans les domaines concernés, au même titre que n'importe quelle autre langue. Ainsi, si la fonction distinctive de l'oudmourte continue d'être liée à l'ethnicité, elle ne joue pas un rôle exclusif de distinction par rapport aux Russes ou aux Tatars, mais d'inclusion dans les domaines artistiques et académiques, en permettant l'adhésion des locuteurs oudmourtes à des groupes qui ne sont pas fondés sur l'ethnicité. Cela a des conséquences: les Oudmourtes présents en tant qu'Oudmourtes dans des sphères artistiques ou universitaires prouvent la capacité de leur culture à exister dans le monde moderne et à être attractive, en théorie au même titre que les cultures dominantes de diffusion internationale.

Enfin, la langue oudmourte peut aussi être utilisée par des personnes qui ne parlent pas oudmourte et ne sont pas oudmourtes, mais s'intéressent aux Oudmourtes pour des raisons politiques ou économiques. On parlera ici de fonction marketing de la langue, issue du pluralisme et de l'entrée de la Russie dans un système économique libéral depuis $1990^{21}$, dans le sens où les acteurs cherchent à satisfaire les besoins des Oudmourtes afin de les rallier à leur cause. Ainsi, dans le domaine commercial, l'utilisation de la langue oudmourte sur les étiquettes des produits ou dans les magasins qui souhaitent la bienvenue aux consommateurs oudmourtes dans leur langue afin de les fidéliser; dans le domaine politique, par exemple, la distribution de livrets de chants et de recettes de cuisine oudmourtes dans les villages par le parti Russie unie pendant les périodes électorales (photo 4).

Photo 4 : «À votre santé, chants oudmourtes, la cuisine de nos peuples ».

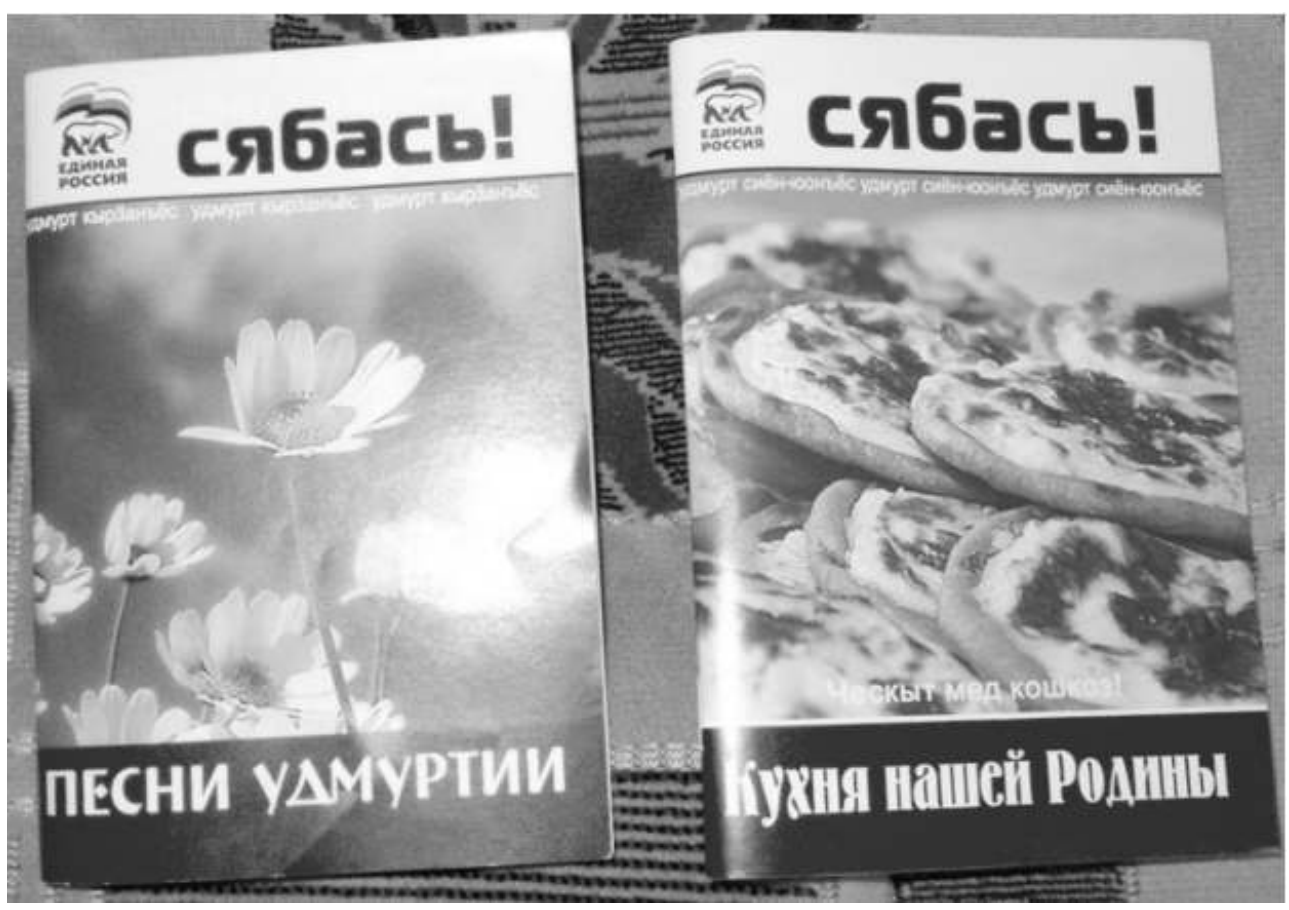

Le logo du parti Russie Unie est visible en haut à gauche des livrets. 

continue de locuteurs, on peut donc nuancer ce constat: l'oudmourte gagne des locuteurs, mais en moins grand nombre que ceux des générations plus âgées qui disparaissent. Les mentalités ont changé : les locuteurs les plus jeunes font preuve d'une bonne capacité d'adaptation au nouveau contexte de globalisation, où la langue oudmourte n'est pas perçue négativement, comme elle l'était pendant la période soviétique par la population russe dominante.

21 À ce stade, il semble que l'obstacle le plus évident à la revitalisation de la langue oudmourte soit l'absence d'engagement de l'État dans les domaines de la revitalisation linguistique et de la protection contre l'assimilation. En effet, en dehors des programmes du ministère de la Politique nationale, aucun projet à grande échelle n'est mené dans la République pour développer l'enseignement ou la pratique de l'oudmourte. D'autre part, l'absence d'initiative visant à appliquer les textes protégeant les minorités de Russie contre l'assimilation linguistique est frappante. Jusqu'à présent, en Ourmourtie, les textes fédéraux ne sont pas appliqués, et les minorités n'ont aucun moyen concret de défendre leurs droits, encore moins de les étendre ${ }^{22}$. Si la revitalisation linguistique est un élément fondamental de la survie et $\mathrm{du}$ développement de la langue, la protection contre l'assimilation en constitue le corollaire indispensable, sans lequel il n'existe aucune garantie de préservation.

\section{BIBLIOGRAPHIE}

CASEN Marie, 2008, Les manifestations de l'identité oudmourte à Iževsk depuis 1985, Mémoire de Master 2, sous la direction d'Eva Toulouze et de Charles URJEWICZ, INALCO, Paris.

CASEN Marie, 2013, "Contemporary Udmurt Ethnic Activity in the context of Udmurt Identity Issues", Journal of Ethnology and Folklore (à paraître).

EDYGAROVA 2012 = ЭДЫГАРОВА СВЕТЛАНА, «ЭТНИЧЕСКИЙ ИДЕНТИТЕТ И ИСПОЛЬЗОВАНИЕ УдМУРТского языкА» (à paraître), Iževsk, Oudmourtie.

GUBOGLO, SMIRNOVA 2002 = ГУБОГЛО М.Н., СМИРНОВА С.К., ФЕНОМЕН УДМУРТИИ. ЭТНОПОЛИТИЧЕСКОЕ РАЗВИТИЕ В КОНТЕКСТЕ ПОСТСОЖЕТСКИЧ ТРАНСФОРМАЦИИ, МОСКВА-ИЖЕВСК: УДМУРТИЯ, p. 424-516. NIКІтІNA 2013 = НИКИТИНА ГАЛИНА АРКАДИЕВНА, « Qui est responsable de la préservation des langues minoritaires? Le cas de la langue oudmourte ", Études finno-ougriennes 45, Paris : ADEFO/ L'Harmattan, p. 82-99.

MARQUARDT Kyle L., 2012, "Stabilization and symbolism: language and regional politics in the Chuvash Republic”, Nationalities Papers: The Journal of Nationalism and Ethnicity no 40:1, pp. 127-147.

ŠABAEV, ŠILOV, DENISENKO 2009 = ШАБАEB У.Р., ШИЛОВ Н.В., ДЕНИСЕНКО В.Н., «ЯЗЫК И ЭТНИЧНОСТЬ. ДИСКУССИИ О ЯЗЫКОВОЙ ПОЛИТИКЕ В РЕГИОНАХ ПРОЖИВАНИЯ ФИННО-УГРОВ», ЭТНОГРАФИЧЕСКОЕ ОБОЗРЕНИЕ $\mathrm{N}^{\circ}$ 2, p. 98, ИЖЕВСК, cité par G. Nikitina, 2013 (cf. plus haut). 
ZAMJATIN, PASANEN, SAARIKIVI 2012 = ЗАМЯТИН КОНСТАНТИН, ПАСАНЕН АННИКА, СААРИКИВИ ЯННЕ, КАК И ЗАЧЕМ СОХРАНЯТЬ ЯЗЫКИ НАРОДОВ РОССИИ? Еd. POGA, The language survival network, Helsinki, 2012.

\section{NOTES}

1. - Consulté le 3 octobre 2013.

2. L'oudmourte est aussi parlé dans les républiques du Bachkortostan, du Tatarstan, de Mari El, dans le Kraï de Perm', les oblasts de Kirov et de Sverdlovsk et, selon le recensement de 2002, dans l'okroug khanty-mansi. Hors de la Russie, on compte des locuteurs au Kazakhstan, en Ukraine, en Estonie, en Hongrie et en Finlande.

3. Site officiel : - Consulté le 29 septembre 2013.

4. Site officiel : - Consulté le 29 septembre 2013.

5. À ce sujet, on peut consulter le quotidien en ligne Susanin : - Consulté le 25 juillet 2013.

6. En pratique, le nombre important d'étudiants oudmourtes dans les filières artistiques (musique, dessin, design) et dans la faculté d'Histoire, ainsi que la présence de professeurs oudmourtes dans ces mêmes filières, rendent possibles les travaux personnels et les mémoires portant sur des aspects de la culture oudmourte.

7. Shudon Korka et Kunnele Kyngyrau : on peut les regarder sur le site de la télévision :- Consulté le $1^{\mathrm{er}}$ octobre 2013.

8. La radio en oudmourte peut être écoutée en direct sur le site de la radio-télévision Moja Udmurtija : - Consulté le 7 octobre 2013.

9. En pratique, des personnes nées de parents oudmourtes, assimilés ou n'ayant pas transmis la langue oudmourte à leurs enfants (ce qui n'est pas rare).

10. L'information m'a été transmise par un des membres de la commission.

11. VKontakte et Facebook.

12. Des propositions sont faites pour traduire un terme russe ou anglais en oudmourte et les internautes ont une quinzaine de jour pour voter pour le néologisme qui leur semble le plus approprié. Celui qui recueille le plus grand nombre de voix est adopté.

13. Udmurtskij každij den'.

14. Ces textes sont polémiques, éducatifs, juridiques, politiques, argumentatifs ou érotiques, pamphlétaires. Ce sont aussi parfois des notices d'utilisation (pour installer un logiciel, par exemple).

15. C'est en particulier un des objectifs de Darali Leli, la rédactrice de Dart, le supplément artistique du quotidien Udmurt Dunne.

16. - Consulté le $1^{\mathrm{er}}$ octobre 2013.

17. À cette même période et lors des rassemblements pour un vote juste à Moscou, des manifestants avaient des pancartes en erza.

18. J'ai constaté à bien des reprises qu'à partir du moment où j'expliquais que j'étais étrangère et en Oudmourtie pour apprendre l'oudmourte, mes interlocuteurs appelaient les employés oudmourtes pour me servir ; ces derniers s'étaient donc identifiés et déclarés comme tels sur leur lieu de travail.

19. Parmi lesquels : Opa val no skal, version oudmourte du Gangnam Style du chanteur coréen Psy, Octe tan iso, imitée du célèbre Ai se eu te pego du brésilien Michel Teló.

20. Lors de mon terrain de juin 2013, j'ai constaté à plusieurs reprises qu'il était difficile pour les jeunes Oudmourtes de langue maternelle oudmourte, qu'ils vivent à la ville ou à la campagne, d'exprimer des sentiments, des idées abstraites ou de lire le journal. 
21. En théorie, l'Oudmourtie est entrée dans l'ère du pluralisme politique et du système économique libéral en 1990. En pratique, les privatisations des principales entreprises ont été effectuées entre 1985 et 1995, et il n'y a pas eu d'autre parti politique organisé que le Parti communiste local, jusqu'en 1995.

22. Comme dans les autres républiques de Russie, et à plus forte raison depuis le début de la présidence de M. Poutine et M. Medvedev, caractérisée par la corruption (de notoriété publique) des services de la Justice.

\section{RÉSUMÉS}

Environ 325000 personnes parlaient oudmourte en 2010, selon le recensement de la population de Russie; cette langue elle a perdu près de $30 \%$ de locuteurs depuis 2002, et autant entre 1989 et 2002. Ces chiffres montrent que l'avenir de la langue oudmourte est en péril, mais ne donnent pas d'information sur les évolutions qu'elle a subies depuis la fin de la période soviétique. Cet article présente un panorama sociolinguistique de l'oudmourte en Oudmourtie durant ces vingttrois dernières années dans ses aspects institutionnels (première partie), mais aussi en tenant compte des pratiques et des comportements des locuteurs actuels (deuxième partie). Les fonctions de la langue oudmourte ont subi des modifications liées à la situation politique, sociale et économique contemporaine. Ces changements sont explorés en troisième partie.

About 325,000 people speak Udmurt in 2010, according to the census of the Russian population. This language has lost nearly $30 \%$ of speakers since 2002 , a loss comparable to the one between 1989 and 2002. These figures show that the future of the Udmurt language is in danger, but do not give information on the changes it has undergone since the end of the Soviet period. This paper presents a sociolinguistic overview of the Udmurt language in Udmurtia during the past 23 years in its institutional aspects (first part), but also taking into account the practices and behaviors of the current speakers (second part). The functions of the Udmurt language have changed related to contemporary political, social and economic situation. These changes are explored in the third part.

\section{INDEX}

nomsmotscles Russes, Tatars, Oudmourtes

Index chronologique : XXIe siècle (début), XXe siècle (fin), XXe siècle, XXIe siècle

Keywords : Udmurt, generation, language census, media, strandardisation, street art, music, Finland, Erzya, Hill Mari, Komi, Meadow Mari, Moksha, Permiak, Russians, Tatars, Hungary, Kazakhstan, Russian Federation, Kirov, Perm', Sverdlovsk, Bashkortostan, Tatarstan, Kazan, Mari-El, Udmurtia, Izhevsk, Alnashi, Malopurga, Mozhga, Uva, Buranovo, Ukraine disciplines erza, komi, mari des plaines, mari des collines, mokcha, oudmourte, permiak Mots-clés : génération, recensement linguistique, médias, standardisation, street art, musique Index géographique : Fédération de Russie, Hanty-Mansijsk, Bachkortostan (République), Tatarstan (République), Kazan, Perm, Iževsk, Alnaši, Malopurga (raïon), Ukraine, Hongrie, Možga, Uva, Buranovo 